\title{
Cochlear Efferent Innervation Is Sparse in Humans and Decreases with Age
}

\author{
Leslie D. Liberman ${ }^{1}$ and ${ }^{\circ} \mathrm{M}$. Charles Liberman ${ }^{1,2}$ \\ ${ }^{1}$ Eaton-Peabody Laboratories, Massachusetts Eye and Ear, Boston, Massachusetts 02114, and ${ }^{2}$ Department of Otolaryngology, Harvard Medical School, \\ Boston, Massachusetts 02115
}

The mammalian cochlea is innervated by two cholinergic feedback systems called the medial olivocochlear (MOC) and lateral olivocochlear (LOC) pathways, which send control signals from the brainstem back to the outer hair cells and auditory-nerve fibers, respectively. Despite countless studies of the cochlear projections of these efferent fibers in animal models, comparable data for humans are almost completely lacking. Here, we immunostained the cochlear sensory epithelium from 23 normal-aging humans (14 males and 9 females), 0 - 86 years of age, with cholinergic markers to quantify the normal density of MOC and LOC projections, and the degree of age-related degeneration. In younger ears, the MOC density peaks in mid-cochlear regions and falls off both apically and basally, whereas the LOC innervation peaks near the apex. In older ears, MOC density decreases dramatically, whereas the LOC density does not. The loss of MOC feedback may contribute to the age-related decrease in word recognition in noise; however, even at its peak, the MOC density is lower than in other mammals, suggesting the MOC pathway is less important for human hearing.

Key words: aging; efferent; hearing

\section{Significance Statement}

The cochlear epithelium and its sensory innervation are modulated by the olivocochlear (OC) efferent pathway. Although the medial OC (MOC) reflex has been extensively studied in humans, via contralateral sound suppression, the cochlear projections of these cholinergic neurons have not been described in humans. Here, we use immunostaining to quantify the MOC projections to outer hair cells and lateral OC (LOC) projections to the inner hair cell area in humans 0 - 89 years of age. We show age-related loss of MOC, but not LOC, innervation, which likely contributes to hearing impairments, and a relative paucity of MOC terminals at all ages, which may account for the relative weakness of the human MOC reflex and the difficulty in demonstrating a robust functional role in human experiments.

\section{Introduction}

The vertebrate inner ear is modulated by cholinergic feedback from the brainstem via the olivocochlear (OC) efferent pathway (Guinan, 2010). The OC system in mammals comprises a few thousand neurons, divided into medial and lateral subdivisions, based on cell body location in the superior olivary complex (Guinan et al., 1983, 1984). Medial OC (MOC) neurons are myelinated and represent the effector arm of a sound-evoked

\footnotetext{
Received Nov. 28, 2018; revised Sept. 27, 2019; accepted 0ct. 5, 2019.

Author contributions: M.C.L. designed research; L.D.L. and M.C.L. performed research; L.D.L. and M.C.L. analyzed data; M.C.L. wrote the paper.

This research was supported by the Lauer Tinnitus Center and by Grants P50-015857 and R01-DC-00188 from the National Institute on Deafness and Other Communication Disorders. We thank Diane Jones for assistance in temporal bone removal. The rhesus cochlear tissue was obtained courtesy of Dr. Troy Hackett and Dr. Ram Ramachandran from the Department of Speech and Hearing Sciences at Vanderbilt University.

The authors declare no competing financial interests.

Correspondence should be addressed to Dr. M. Charles Liberman at Charles_Liberman@meei.harvard.edu.

https://doi.org/10.1523/JNEUROSCI.3004-18.2019

Copyright $\odot 2019$ the authors
}

negative-feedback loop (Liberman and Brown, 1986) that projects to cochlear outer hair cells (OHCs), as schematized in Figure 1. MOC activity diminishes cochlear sensitivity (Wiederhold and Kiang, 1970) by reducing the normal contribution of OHC electromotility to amplification of mechanical vibration of the sensory epithelium (Russell and Murugasu, 1997). The lateral OC (LOC) neurons are unmyelinated and project to dendrites of auditorynerve fibers (Fig. 1) near their synapses with inner hair cells (IHCs; Liberman, 1980), where they modulate the excitability of these sensory neurons (Groff and Liberman, 2003). It is not clear whether LOC neurons are activated (or suppressed) by sound.

Several functional roles have been suggested for these feedback systems. MOC feedback extends the dynamic range of the ear and increases the detectability of signals in noise (Kawase et al., 1993). The LOC system balances neural outputs from the two ears, as needed for localization of high-frequency sounds based on interaural level differences (Darrow et al., 2006). Both MOC and LOC systems protect the ear from acoustic overstimulation ( $\mathrm{Ra}-$ jan, 1988; Kujawa and Liberman, 1997; Darrow et al., 2007), and 


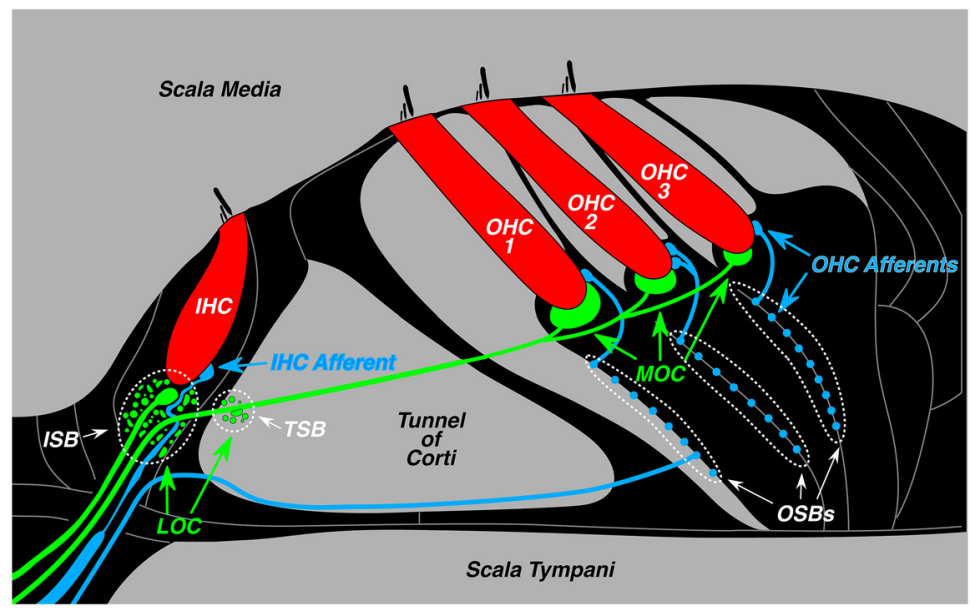

Figure 1. Schematic illustration of the organ of Corti showing the afferent and efferent innervation of the IHCsand $\mathrm{OHCs}$, including both the lateral and medial divisions of the $0 \mathrm{C}$ feedback system. Efferents in the IHC area spiral in two bundles, the inner spiral bundle (ISB) and the tunnel spiral bundle (TSB), while afferents underneath the $\mathrm{OHCs}$ spiral in the outer spiral bundles (OSBS).

both are necessary for normal cochlear development (Walsh et al., 1998).

Neuroanatomy, neurophysiology, synaptic transmission, and peripheral effects of MOC and LOC systems have been extensively studied in animals (Guinan, 2010). However, in humans, only the peripheral effects of the MOC system have been well characterized. Because the MOC reflex is binaural, contralateral sound can activate MOC fibers to the ipsilateral ear and suppress responses such as otoacoustic emissions (Collet et al., 1990). Studies of "contra-sound suppression" suggest that MOC reflex strength declines with age (Kim et al., 2002; Jacobson et al., 2003; Konomi et al., 2014; Lisowska et al., 2014). Given the hypothesized role in the control of masking, this reflex decline may contribute to problems with hearing in noise in the aging ear. However, based on the contra-sound suppression assay, the MOC reflex in humans is significantly weaker than in other laboratory mammals (e.g., mice; Chambers et al., 2012).

In humans, the understanding of OC neuroanatomy is fragmentary. The organization of LOC versus MOC subdivisions in the brainstem has been verified (Moore et al., 1999), and the presence of cholinergic fibers and terminals in the cochlea has been documented (Ishii et al., 1967; Nomura, 1976; Schrott-Fischer et al., 1994, 2007). Electron microscopy studies show cochlear efferent synapses that resemble those in animals (Nadol, 1983a,b). However, no study has quantified the normal density of efferent innervation along the cochlear frequency (spiral) axis or the age-related changes in these projections.

Here we address these fundamental issues with a quantitative confocal study of immunostained inner ears from 23 "normalaging" subjects (i.e., individuals without exposure to known ototoxic substances or excessive noise exposure), ranging in age from a few weeks to 86 years. Results show different frequency distributions for LOC versus MOC projections in the younger ears, and an age-related loss of MOC innervation, without loss of LOC projections. Results also suggest that the MOC innervation is relatively sparse in humans compared with mouse, guinea pig, and rhesus macaque, which helps to explain why the human MOC reflex is so weak compared with other mammals studied.

\section{Materials and Methods}

Subjects and groups. The materials for the present study are human temporal bones obtained at autopsy. The subject pool included 23 ears from
23 individuals, ranging in age from neonatal to 86 years. The majority ( 21 of 23 individuals) were also studied in a prior report on agerelated primary degeneration of auditorynerve fibers (Wu et al., 2019). None of these cases had any explicit history of otologic disease other than age-related hearing loss, which was noted in the medical record for three case patients, who were 78,80 , and 86 years of age. Two of these three case patients had audiograms: both showed thresholds within $10 \mathrm{~dB}$ of the median better-ear threshold for age- and sex-matched subjects (Gordon-Salant, 2005). One of the youngest subjects (age, 24 years) had an audiogram documenting normal thresholds at all standard frequencies. All procedures concerning the handling of human tissues were approved by the Human Studies Committee of Massachusetts Eye and Ear. For comparison of the density of efferent innervation, four ears from each of three other species (mouse, guinea pig, and rhesus macaque) were also processed and imaged in identical fashion. Mice were male $\mathrm{CBA} / \mathrm{CaJ}$ mice (age, 6.5 weeks), guinea pigs were male and female Hartley strain (age, 6.5 weeks), and monkeys were male (age, 9.5 and 11.5 years). Mouse and guinea pig tissue was extracted at Massachusetts Eye and Ear under protocols approved by the institutional Animal Care Committee. Rhesus tissue was extracted at Vanderbilt University (Nashville, TN) under protocols led by Dr. Ram Ramachandran and approved by the Institutional Animal Care and Use Committee of Vanderbilt University.

Cochlear processing. The human temporal bones were extracted with a bone-plugging tool (Schuknecht, 1993), from 5 to $13 \mathrm{~h}$ after death (median, $9.0 \mathrm{~h}$ ) and immersed in buffered 10\% formalin; round and oval windows were opened, and was formalin flushed through the scalae. After postfixation $\left(4^{\circ} \mathrm{C}\right)$ for $\geq 6 \mathrm{~d}$, the bone plug containing the cochlea was drilled to remove much of the petrous bone and then immersed in decalcifying solution (EDTA) at room temperature for $\sim 4$ weeks. Cochleas from mice, guinea pigs, and monkeys were fixed by intravascular and cochlear perfusion of buffered $4 \%$ paraformaldehyde, and then postfixed for $2 \mathrm{~h}$. Cochleas were microdissected into $6-12$ pieces (depending on species), each containing the osseous spiral lamina and the attached organ of Corti. Following immunostaining, the cochlear spiral was mapped onto low-power images of the microdissected pieces by tracing an arc along the tunnel of Corti, after which, normalized cochlear length was converted to frequency. For humans, this conversion was based on a Greenwood function (Greenwood, 1990) modified to produce best frequencies at the apex and base of the cochlea of $100 \mathrm{~Hz}$ and $20 \mathrm{kHz}$, respectively. Cochlear frequency maps for mouse (Taberner and Liberman, 2005), guinea pig (Tsuji and Liberman, 1997), and monkey (Valero et al., 2017) have been described previously. In humans, 14 frequency locations from 0.175 to $16 \mathrm{kHz}$ were calculated, and superimposed on the low-power images of the microdissected pieces, at half-octave intervals along the length of the spiral, to pinpoint the image acquisition loci in each case. In each of the other species, images were also acquired at several log-spaced frequency locations.

Immunostaining protocols. Cochlear pieces were permeabilized with a freeze/thaw step in 30\% sucrose, followed by $1 \mathrm{~h}$ at room temperature in a blocking buffer (PBS with 5\% normal horse serum and $0.3-1 \%$ Triton $\mathrm{X}-100$ ). Tissue was then incubated overnight at $37^{\circ} \mathrm{C}$ with some combination of the following primary antibodies (plus $0.3-1 \%$ Triton $\mathrm{X}-100$ ): (1) chicken anti-neurofilament (catalog \#AB5539, Millipore Bioscience Research Reagents) at 1:1000 to visualize nerve axons; (2) rabbit antimyosin VI and/or VIIa (\#25-6791 and 25-6790, respectively, Proteus Biosciences) at 1:100 to count hair cells; and (3) goat anti-choline acetyltransferase (ChAT; catalog \#AB144P, Millipore) at 1:100 to quantify efferent projections from the olivocochlear bundle. Primary incubations were followed by two sequential $60 \mathrm{~min}$ incubations at $37^{\circ} \mathrm{C}$ in speciesappropriate secondary antibodies (coupled to Alexa Fluor dyes) with 

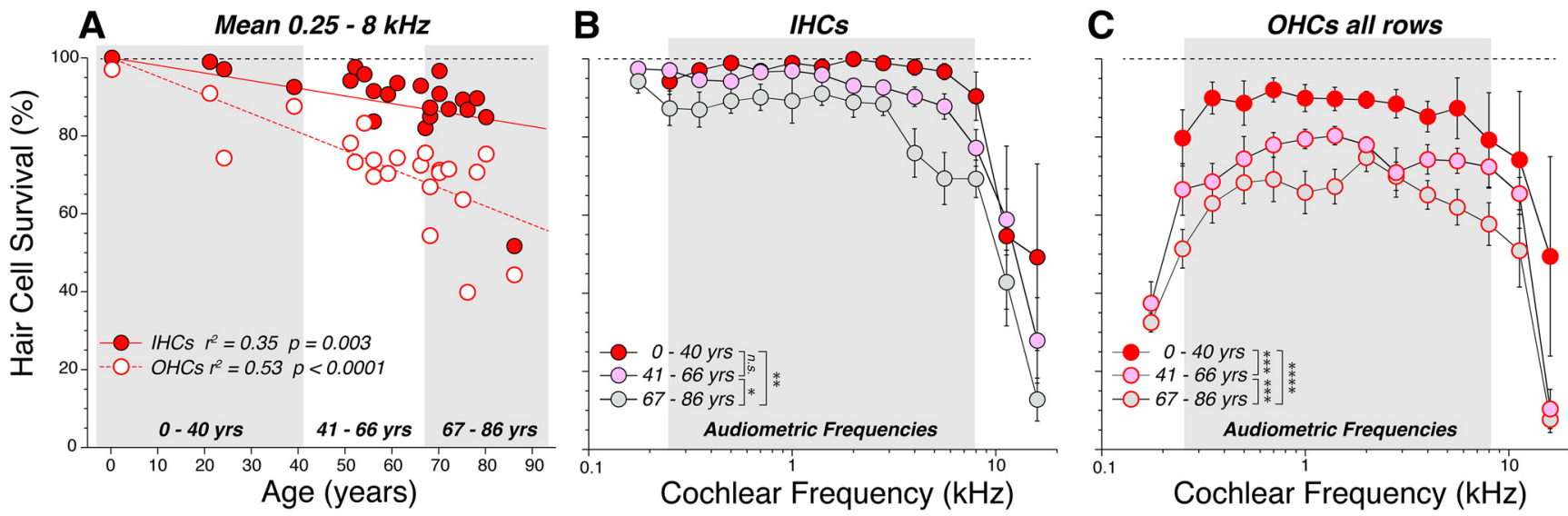

Figure 2. Patterns of hair cell loss in the 23 human temporal bones analyzed in the present study. $\boldsymbol{A}$, Age-related loss of hair cells, showing mean survival in each case, averaged over the 11 half-octave samples within the audiometric frequency range ( $0.25-8.0 \mathrm{kHz}$ inclusive). $\mathrm{OHC}$ values represent the average of all three rows. Best-fit straight lines are shown in red, as indicated in the key, along with correlation coefficients and $p$ values. $\boldsymbol{B}, \boldsymbol{C}$, Frequency pattern of hair cell loss, showing mean survival ( \pm SEMs) of $\mathrm{IHCs}(\boldsymbol{B})$ and $0 \mathrm{HCs}(\boldsymbol{C})$ at each of the 14 half-octave cochlear frequency locations sampled. Case patients are arbitrarily divided into three age groups, as illustrated in $\boldsymbol{A}$. The significance of the intergroup differences is indicated by asterisks: ${ }^{*} p<0.05$; ** $p<0.005$; ${ }^{* * *} p<0.0005 ; * * * * 0.0001$; or n.s. (not significant).

A
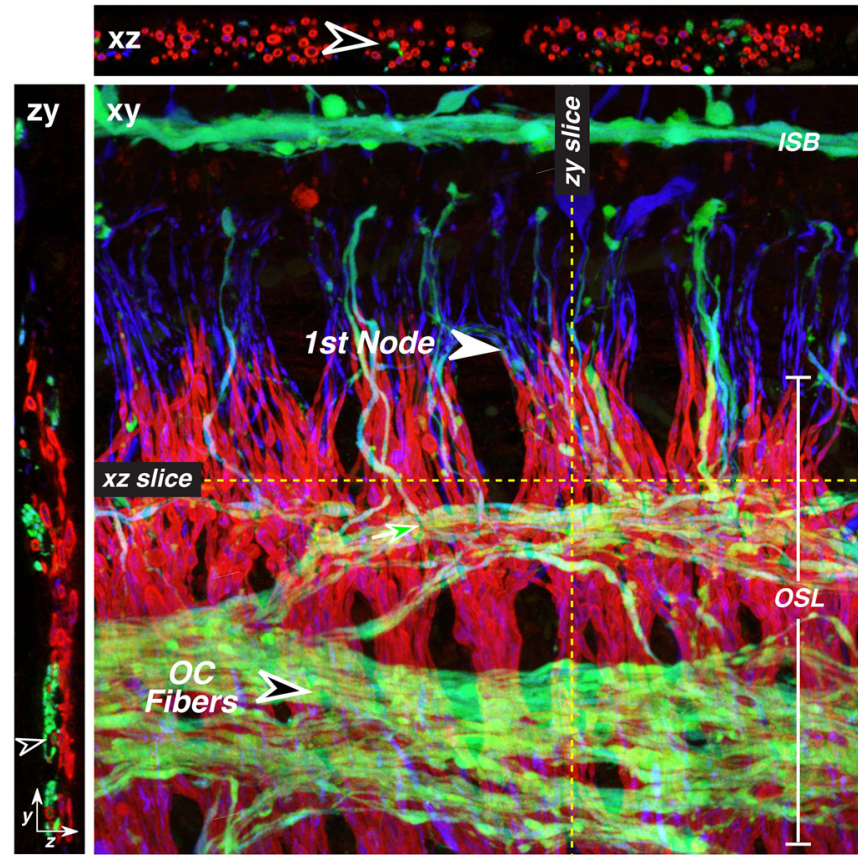

B

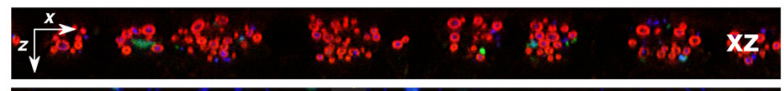

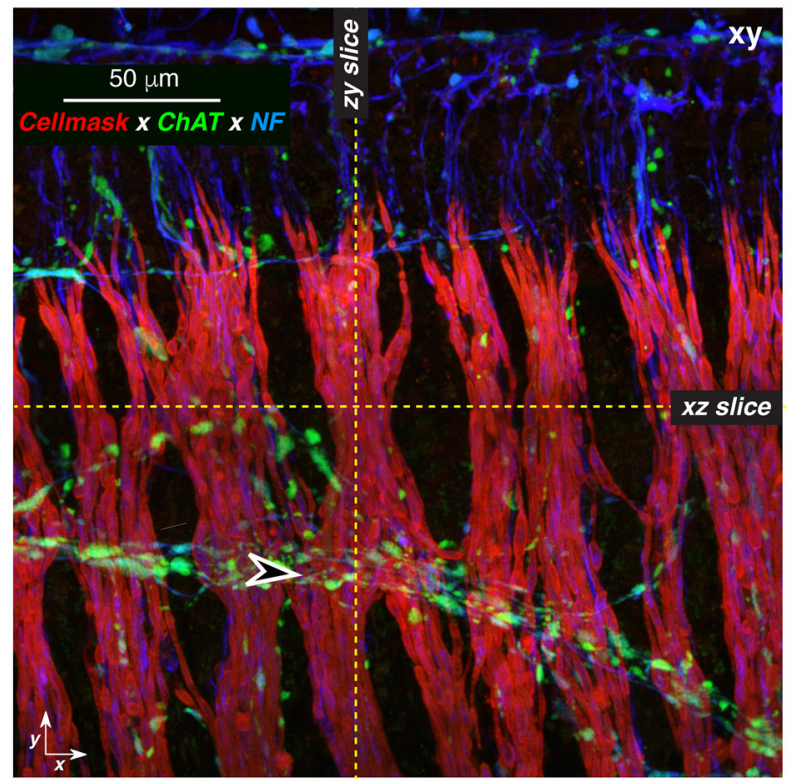

Figure 3. Most of the ChAT-positive fibers in the osseous spiral lamina are unmyelinated. $\boldsymbol{A}, \boldsymbol{B}$, These images from two confocal $z$-stacks are both from a 78 -year-old male. In each cochlear frequency region $(\boldsymbol{A}, 0.35 \mathrm{kHz} ; \boldsymbol{B}, 4.0 \mathrm{kHz})$, a maximum projection in the $x y$ plane is shown, along with selected $x z$ and $z y$ slices, positioned as indicated by the dashed yellow lines. Black-filled arrowheads in both xy projections point to the spiraling bundles of efferent fibers. Arrowhead in $A$ zy points to a spiraling myelinated efferent (ChAT-positive) axon, while the arrowhead in $A$ xz points to the unmyelinated radially directed portion of an efferent axon. Scale bar and orientation arrows in $\boldsymbol{B}$ apply to $\boldsymbol{A}$. The $x$ arrows point along the spiral toward the base, $y$ arrows point radially toward the organ of Corti, and $z$ arrows point toward scala tympani. NF, neurofilament.

0.3-1\% Triton X-100. After immunostaining, all pieces from each cochlea were slide mounted in Vectashield, and coverslipped, and the coverslip was sealed with nail polish. After the analysis of hair cells and efferent terminals, the coverslips were removed and the tissue was incubated in a fluorescent membrane dye (CellMask Orange, catalog \#C10045, Thermo Fisher Scientific) at 1:5000 with $0.3 \%$ Triton X-100 for $5 \mathrm{~min}$ to label the myelin sheaths. The pieces were remounted and coverslipped with Vectashield. This final treatment did not disturb the preexisting immunostains.

Hair cell counts. For all stained tissues, confocal $z$-stacks were acquired at equally spaced locations along the spiral, with $0.33 \mu \mathrm{m} z$-spacing on a
Leica SP8 using a $63 \times$ glycerol objective (1.3 numerical aperture). At each cochlear location, an image stack of IHCs and OHCs spanning $\sim 250 \mu \mathrm{m}$ of cochlear length was acquired. In each stack, hair cell fractional survival was quantified by counting the number of remaining cells in each row as the numerator and estimating the number of missing cells to include in the denominator. In case patients with only scattered loss, missing cells are easy to count as gaps in the regular array. In case patients with more massive losses, the denominator was estimated from the minimal loss case patients, using mean values as a function of cochlear location. Among the OHCs, the denominator was set by the first row of cells, because they are always the most regularly arrayed. 
Innervation analysis. To estimate the innervation density of the LOC and MOC projections, we acquired high-power images of the inner and outer hair cell areas, respectively. In each cochlear location, and separately for inner and outer hair cell areas, we acquired two adjacent $z$-stacks (each spanning $112 \mu \mathrm{m}$ of the cochlear spiral), taking care to span, along the $z$-axis, the entire region containing ChAT-positive terminals or fibers. The ChAT channel of each $z$-stack maximum projection was extracted and ported to ImageJ, where the default auto-thresholding algorithm was used to count the total signal in the projection (https:// imagej.net/Auto_Threshold\#Default), which was expressed as the number of suprathreshold pixels. This algorithm requires no user input: it divides the entire image into object versus background by taking an initial threshold, computing averages of the pixel values equal to or greater than threshold and those less than threshold, then incrementing the threshold value until it is larger than the composite average.

In prior studies, we have compared several alternate approaches to the quantification of MOC innervation density (i.e., counting of tunnelcrossing fascicles or measuring their summed diameter; Liberman and Gao, 1995; Liberman et al., 2000), counting the average number of MOC terminals contacting each OHC (Liberman et al., 1990, 2014), or measuring the projected silhouette areas, either manually (Maison et al., 2003) or automatically (Yin et al., 2014). In prior work, we have shown (1) that the fascicle diameters in the tunnel are highly correlated with silhouette areas of terminals under the OHCs (Liberman and Gao, 1995), (2) that immunostained silhouettes are highly correlated with maximum terminal areas extracted from serial-section electron microscopy (Liberman et al., 1990), and (3) that terminal counts are highly correlated with silhouette areas (M. C. Liberman, unpublished observations). Here, we chose to use automatic measurement of immunostained silhouette areas, (1) because it is completely computer driven and thus not subject to user bias; and (2) because so many of the MOC terminals were small en passant swellings, it was difficult to set a reproducible criterion for their enumeration.

Experimental design and statistical analysis. This was a prospective study of autopsy case patients of either sex from the Massachusetts General Hospital, meeting the following inclusion criteria: temporal bones were extracted at a postmortem time of $<13 \mathrm{~h}$, and there was no medical history of otological disease. Statistical analysis was performed in GraphPad Prism version 8.2.1. Age-related changes (Fig. $2 A$; see Figs. 5A, $7 A$, $9 B$ ) were assessed by linear regression, and intergroup comparisons (Fig. $2 B, C$; see Figs. $5 B, C, 7 B, C$ ) were assessed by two-way ANOVA followed by a Holm-Sidak multiple-comparisons test.

\section{Results}

\section{Age-related loss of hair cells}

The human cochlea contains a sensory epithelium, the organ of Corti (Fig. 1), that spirals for $\sim 35 \mathrm{~mm}$ from the high-frequency basal tip to the low-frequency apex. This epithelium normally contains one row of $\sim 3500$ IHCs, three rows each containing $\sim 4200 \mathrm{OHCs}$, and an occasional fourth row of OHCs. For each of the 23 temporal bones in the present study, the sensory epithelium was microdissected and immunostained. Hair cell counts and the analysis of afferent and efferent innervation was performed on confocal $z$-stacks corresponding to half-octave intervals of cochlear frequency from 0.175 to $16 \mathrm{kHz}$. The fractional survival of hair cells is summarized in Figure 2. Age-related loss of OHCs outpaces the age-related loss of IHCs by $>2: 1$ (Fig. $2 A$ ): $4.5 \%$ loss per decade for OHCs versus $2.0 \%$ loss per decade for IHCs. Although, with increasing age, scattered hair cell loss is seen throughout the cochlear spiral for both IHCs and OHCs, the loss is most dramatic for IHCs in the extreme base (Fig. 2B) and in both the basal and apical extremes among the OHCs (Fig. 2C).

\section{MOC innervation and age-related loss}

MOC neurons in mammals project from cell bodies in brainstem regions near the medial superior olive, and send myelinated axons to the OHC region, both ipsilaterally and contralaterally,
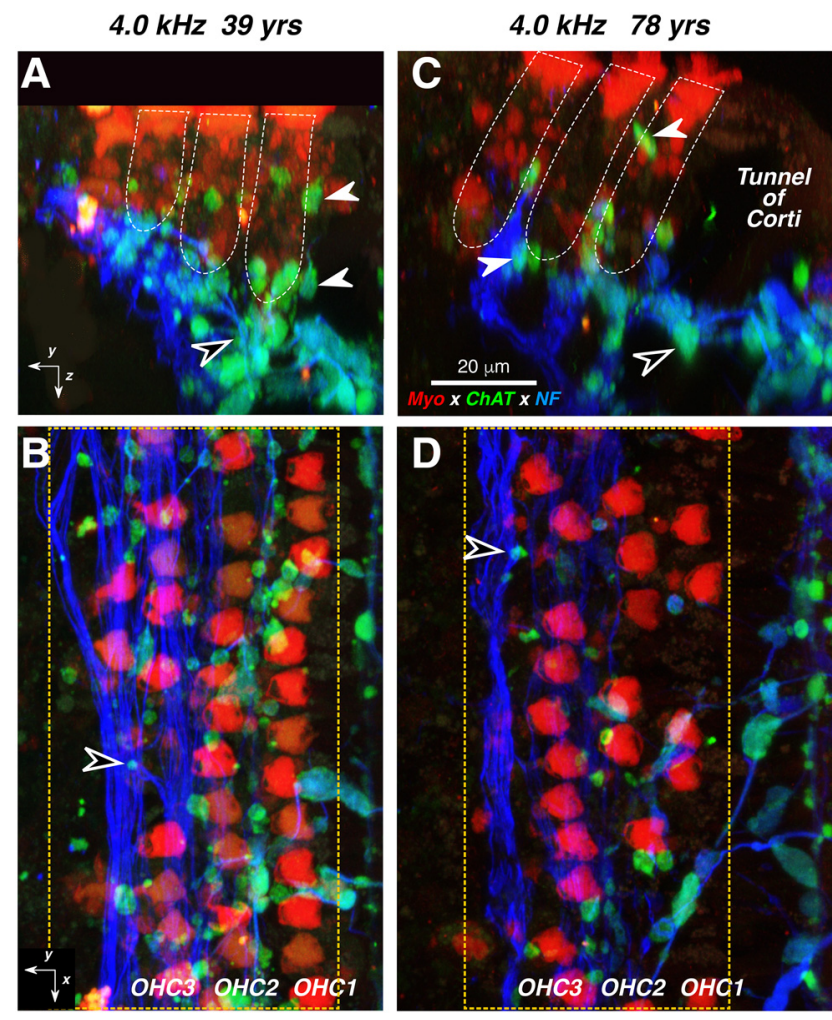

Figure 4. $\boldsymbol{A}-\boldsymbol{D}$, Confocal images of the MOC innervation of $\mathrm{OHC}$ in a middle-aged $(\boldsymbol{A}, \boldsymbol{B})$ vs an older $(\boldsymbol{C}, \boldsymbol{D})$ subject. From each subject, two views of the same confocal $z$-stack are shown: $\boldsymbol{B}$ and $\boldsymbol{D}$ are maximum projections in the acquisition ( $x y)$ plane, while $\boldsymbol{A}$ and $\boldsymbol{C}$ are maximum projections of the entire stacks in the zy plane (i.e., rotated $90^{\circ}$ to show the view along the axis of the cochlear spiral) with the approximate boundaries of the three $\mathrm{OHC}$ rows indicated in white dashed lines. White arrowheads in $\boldsymbol{A}$ and $\boldsymbol{C}$ point to $\mathrm{MOC}$ terminals at the bases, or along the sides of $\mathrm{OHCS}$, while black-filled arrowheads point to $\mathrm{MOC}$ terminals within the spiraling bundles of type II fibers (e.g., at the arrowheads in $\boldsymbol{B}$ and $\boldsymbol{D}$ ). Immunostaining key and scale bar in $\boldsymbol{C}$ apply to all panels. Orientation arrows in $\boldsymbol{A}$ and $\boldsymbol{B}$ apply to $\boldsymbol{C}$ and $\boldsymbol{D}$, respectively. The $x$ arrows point along the spiral toward the apex, $y$ arrows point radially away from the modiolus, and $z$ arrows point toward scala tympani. Dashed yellow boxes enclose the regions included in the digitizations used to quantify $\mathrm{MOC}$ terminal density without including tunnel-crossing fascicles. yrs, Years; NF, neurofilament; Myo, myosin Vlla.

where they terminate on the OHCs (Fig. 1) as well as on the spiraling dendrites of the sensory (afferent) innervation of OHCs, the type II spiral ganglion neurons (Liberman and Brown, 1986; Thiers et al., 2002).

Since the MOC system is predominately cholinergic, those projection patterns are well seen in the distribution of ChATimmunostained fibers and boutons in the osseous spiral lamina (Fig. 3) and organ of Corti (Fig. 4). In the osseous spiral lamina, efferent fibers take a spiral course within a series of bundles traveling orthogonally to the radially oriented auditory-nerve fibers, riding on the scala-vestibuli side of the sensory fascicles (Fig. 3, zy projections). As they approach the organ of Corti, the efferents turn radially to join the afferents. In this region, which is close to the distal ends of these fibers in the organ of Corti, very few of the ChAT-positive efferents appear myelinated (i.e., surrounded by a cell mask-positive sheath; Fig. $3 A, B, x z$ projections).

The MOC terminal boutons in the organ of Corti (Fig. 4) are especially well seen in the reprojection of the image stacks into the $z y$ plane (Fig. $4 A, C$ ), where the sensory epithelium is viewed as if in a thick cross section. MOC boutons (Fig. $4 A, C$, white-filled arrows) can be seen on the basal pole and along the sides of the hair cells (Fig. 4A, C, stained with anti-myosin VIIa; red) as well 

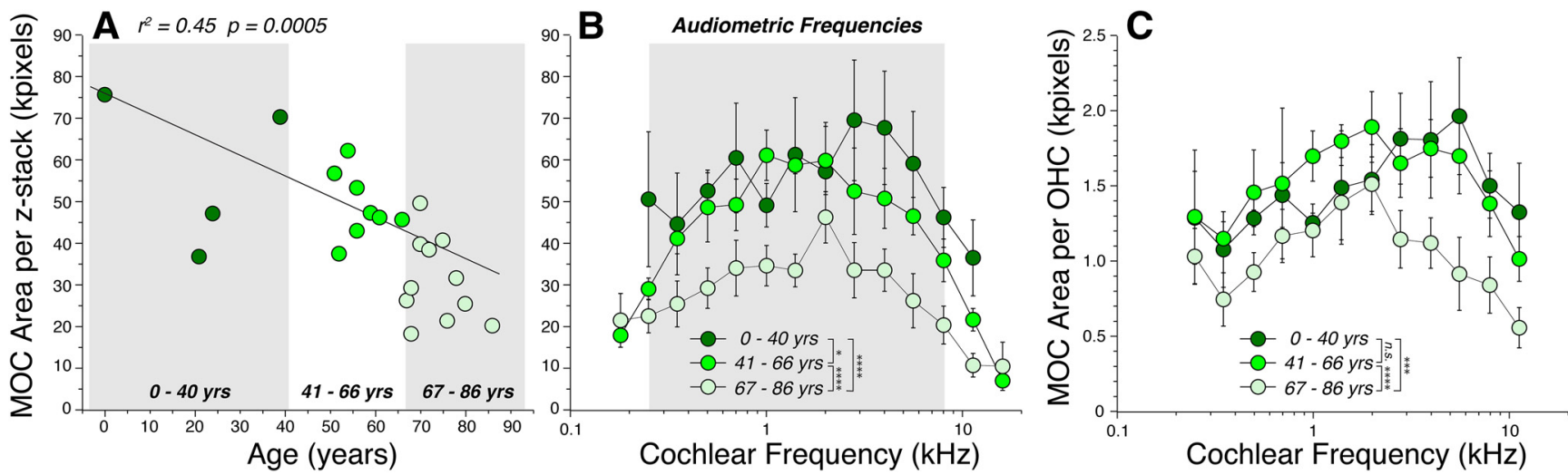

Figure 5. MOC innervation density decreases significantly with age. $A$, Each point shows the mean area of the ChAT-positive boutons in the $0 H C$ area, averaged over 11 half-octave samples within the audiometric frequency range $(0.25-8.0 \mathrm{kHz}$ inclusive) in each case. The correlation coefficient for the linear regression is shown along with the $p$ value. $\boldsymbol{B}, \boldsymbol{C}$, Frequency pattern of MOC innervation density, showing mean values $( \pm$ SEMs), for case patients arbitrarily divided into three age groups, as shown by the gray boxes in $A$. In all panels, MOC area is expressed in kilopixels, as extracted from maximum projections in the xy plane, such as those in Figure $4, \boldsymbol{B}$ and $\boldsymbol{D}$. The data in $\boldsymbol{C}$ are the same as those in $\boldsymbol{B}$, except they have been normalized by dividing by the number of surviving $0 \mathrm{HC}$ in the same $z$-stack. Significance of the intergroup differences are indicated by asterisks, or n.S., as described for Figure 2: precise $p$ values are in the text.

as among the type II dendrites (Fig. $4 A, C$, stained with antineurofilament; blue), which spiral between the supporting cells in the region underneath the OHCs, as shown by the black-filled arrow in Figure 3, $A$ and $C$. A radial gradient is suggested by the images in Figure 4 (i.e., the innervation density is highest among the first-row OHCs and lowest among the third- and fourth-row OHCs). An age-related loss of MOC innervation density is suggested by the difference in the numbers of ChAT-positive boutons in the $4.0 \mathrm{kHz}$ region of the 78-year-old subject (Fig. 4C,D) compared with the same region in the 39 -year-old subject (Fig. $4 A, B)$.

To quantify the density of MOC innervation as a function of cochlear location and age, we took confocal $x y$ projections, like those in Figure 4, $B$ and $D$; extracted the ChAT channel; applied an auto-thresholding algorithm in ImageJ; and averaged the resultant total silhouette area in each projection across cases and at each cochlear location. Several important trends are visible in the results, as summarized in Figure 5.

First, in the youngest ears $(0-40$ years of age; Fig. $5 B)$, the density peaks in the $4 \mathrm{kHz}$ region (upper basal turn) and falls off slowly toward the apex, and more rapidly toward the base. A similar pattern (i.e., peak density occurring in the upper basal turn of the cochlea) has been observed in other low-frequency mammals such as cat and guinea pig (Liberman et al., 1990; Liberman and Gao, 1995).

Second, the MOC density decreases with age. When data from each case are averaged across all audiometric frequencies $(0.25$ $8.0 \mathrm{kHz}$ inclusive) and plotted versus age (Fig. $5 \mathrm{~A}$ ), the linear regression is highly significant $\left(r^{2}=0.45, p=0.0005\right)$. The agerelated differences are greatest in the high-frequency regions (Fig. $5 B$ ), where the mean $\mathrm{MOC}$ areas in the oldest group are less than half of those in the youngest group. Two-way ANOVAs suggest significant pairwise differences between all groups (young vs middle, $p=0.36$; young vs old, $p<0.0001$; middle vs old, $p<$ $0.0001)$.

Last, the age-related loss of MOC density is not simply due to the age-related loss of peripheral targets (i.e., OHCs). As shown in Figure $5 C$, the age-related loss of MOC terminals is still significant when the density is normalized to the numbers of surviving OHCs in the same $z$-stacks ( $p=0.0002$ for young vs old groups; $p<0.0001$ for middle vs old groups; young vs middle are statistically indistinguishable, $p=0.60$ ).

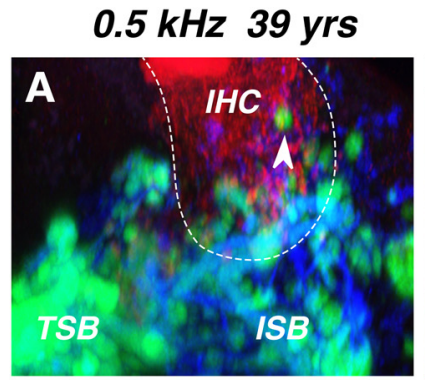

\section{$4.0 \mathrm{kHz} 39 \mathrm{yrs}$}
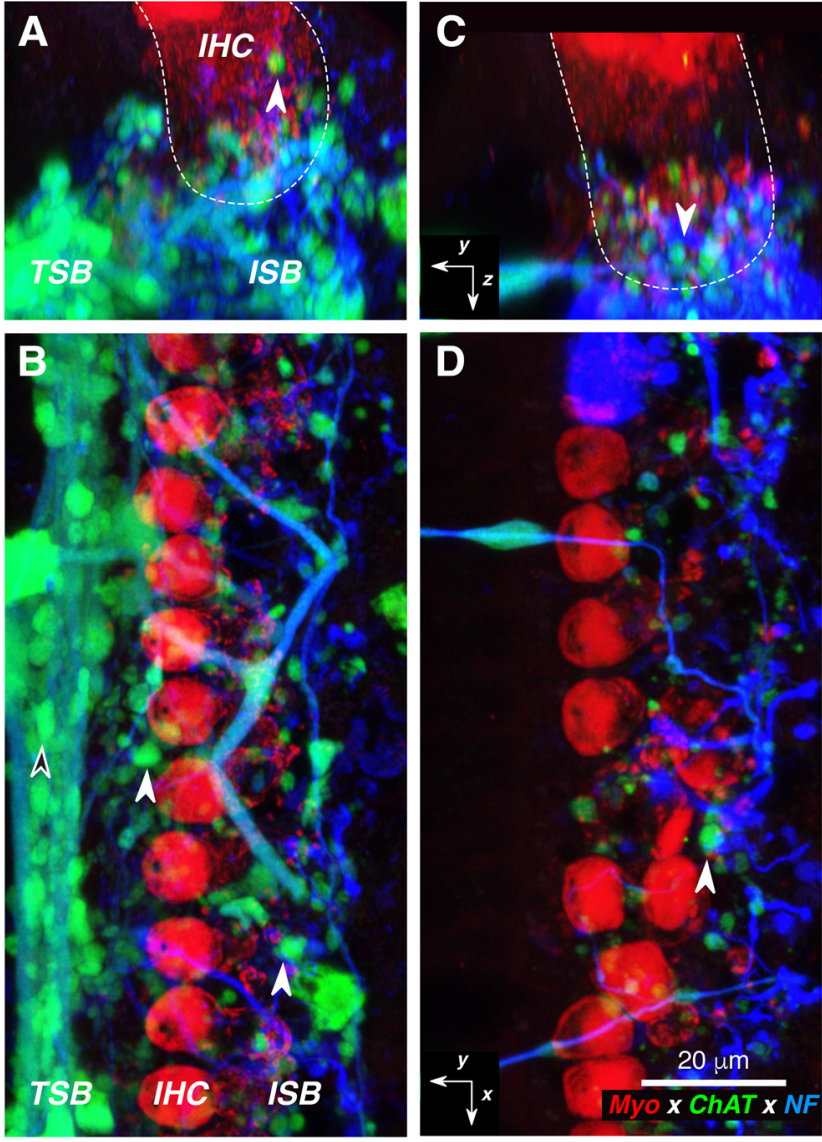

Figure 6. $A-D$, Confocal images of the $L O C$ innervation of IHCs from the apical $(A, B)$ vs basal $(C, D)$ half of the cochlea from one subject. At each locus, two views of the same confocal $z$-stack are shown: $\boldsymbol{B}$ and $\boldsymbol{D}$ are maximum projections in the acquisition plane $(x y)$, while $\boldsymbol{A}$ and $\boldsymbol{C}$ are maximum projections of the entire stacks in the zy plane, with the approximate boundaries of the IHCs indicated in white dashed lines. White-filled arrowhead in all panels point to ChATpositive boutons in the ISB, while the black-filled arrowhead in $\boldsymbol{B}$ points to a ChAT-positive bouton in the TSB. Immunostaining key and scale bar in $\boldsymbol{D}$ apply to all panels, and the orientation arrows in $\boldsymbol{C}$ and $\boldsymbol{D}$ also apply to $\boldsymbol{A}$ and $\boldsymbol{B}$, respectively: the $x$ arrows point along the spiral toward the apex, $y$ arrows point radially away from the modiolus, and $z$ arrows point toward scala tympani. yrs, Years; NF, neurofilament. 

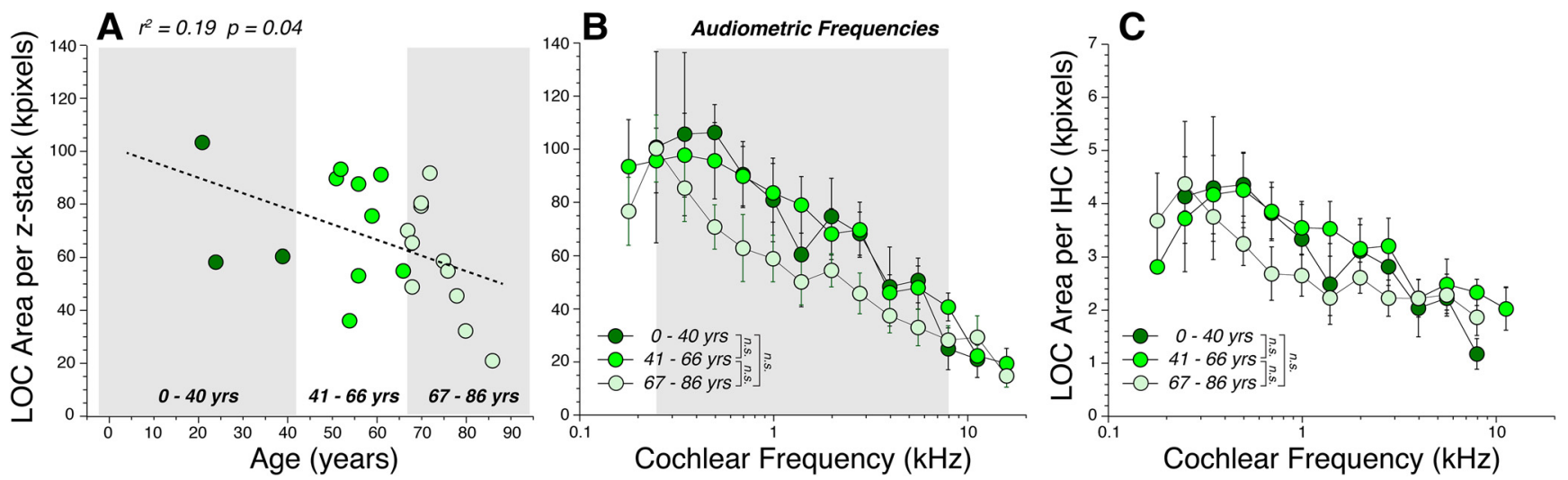

Figure 7. LOC innervation density decreases with age, but not after correction for the loss of IHCs. $A$, Each point shows the mean area of the ChAT-positive boutons in the IHC area, averaged over 11 half-octave samples within the audiometric frequency range $(0.25-8.0 \mathrm{kHz}$ inclusive) in each case. The correlation coefficient and $p$ value for the linear regression is shown. $\boldsymbol{B}$, Frequency pattern of LOC area measures, showing mean values ( \pm SEMs), for cases arbitrarily divided into three age groups, as shown by the gray boxes in $\boldsymbol{A}$. $C$, Same data as in $\boldsymbol{B}$, except the areas have been divided by the number of surviving IHCs in the samez-stacks. In all panels, LOC area is expressed in kilopixels, as extracted from maximum projections in the $x y$ plane, such as those in Figures $3 \boldsymbol{B}$ and $2 \boldsymbol{D}$. The ChAT immunostaining in LOC area for the youngest subject ( 0.1 years) was too dim and indistinct to be reliably measured. The intergroup differences are not statistically significant (n.s.): precise $p$ values are in the text.
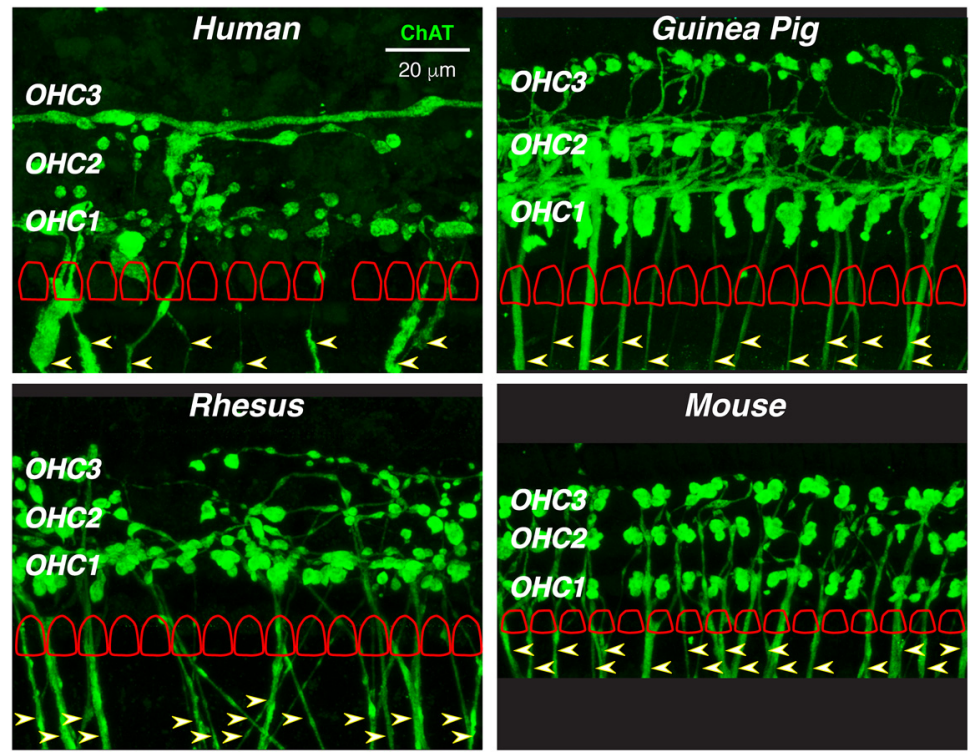

Figure 8. In humans, the MOC innervation is less dense than in other mammals. Each micrograph is the maximum projection from the ChAT channel of a z-stack taken from the cochlear region of maximum innervation density in each of four species: human ( $4.0 \mathrm{kHz}$ region of a 39 -year-old female), rhesus macaque ( $2.0 \mathrm{kHz}$ region of a 9-year-old male), guinea pig (4.0 kHz region of a 1.5 -month-old female), and mouse ( $22.6 \mathrm{kHz}$ region of a 1.5 -month-old male). In each image, the locations of the three $\mathrm{OHC}$ rows are indicated, and the outlines of the first-row $\mathrm{OHCs}$ (cuticular plates) are schematized (to scale, but regularized and offset vertically for clarity) as extracted from the same z-stack. In each image, tunnel-crossing efferent bundles are noted by arrowheads: in the mouse image, the tunnel-crossing bundles are truncated, because the original image field was more restricted than the others. The scale bar in the human panel applies to all images.

\section{LOC innervation and age-related loss}

LOC neurons in the mammal project via unmyelinated axons from the region in and/or around the lateral superior olive, primarily to the ipsilateral cochlea. Throughout much of their course from brainstem to cochlea, they are intermingled with MOC axons, including in the spiraling bundles within the osseous spiral lamina (Fig. 3). Within the organ of Corti, each peripheral LOC axon continues to spiral in the inner spiral bundle (ISB), directly underneath the IHCs and/or in the tunnel spiral bundle (TSB; Fig. 1). As they spiral, they send off terminal branches and make en passant synapses with their peripheral targets. These targets include (1) primarily the unmyelinated terminals of (afferent) auditory-nerve fibers innervating IHCs as well as (2) the IHCs themselves, (3) the afferent auditory-nerve fibers contacting OHCs, and (4) the MOC fibers as they pass through the tunnel spiral bundle on their way to the OHCs (Liberman, 1980).

As suggested by the confocal images in Figure 6, there is an apical-basal gradient of innervation density for the LOC system. These trends were quantified by measuring silhouette areas in the ChAT channel, exactly as for the MOC system. As shown in Figure $7 B$, the peak density for all age groups is at a much more apical (lower frequency) cochlear locus than the MOC system (Fig. 5). The age-related changes were less dramatic than for the MOC system: the relation between age and LOC density, as averaged across all audiometric frequencies, was borderline significant (Fig. $7 B ; p=0.04$ ). When considering the apical-basal LOC distributions (Fig. $7 B$ ), all the age groups were statistically indistinguishable (young vs middle, $p=0.85$; young vs old, $p=0.10$; middle vs old, $p=0.051)$. After adjusting for the age-related loss of IHCs (Fig. 2A), and thus, by extension, for the loss of their peripheral targets (the auditory-nerve fibers), intergroup differences still were not significant (Fig. $7 C$; young vs middle, $p=0.77$; young vs old, $p=$ 0.62 ; middle vs old, $p=0.79$ ).

\section{Interspecies comparisons of MOC and LOC projections}

Qualitative comparisons to prior cholinergic immunostaining studies of cochleas from cat (Liberman et al., 1990), mouse (Liberman et al., 2014), guinea pig (Liberman and Gao, 1995), or ferret (Irving et al., 2011) suggest that the MOC system is less robust in humans than in other well studied laboratory animals. To make the comparison more quantitative, we prepared new material from mouse, guinea pig, and rhesus macaque, using the 

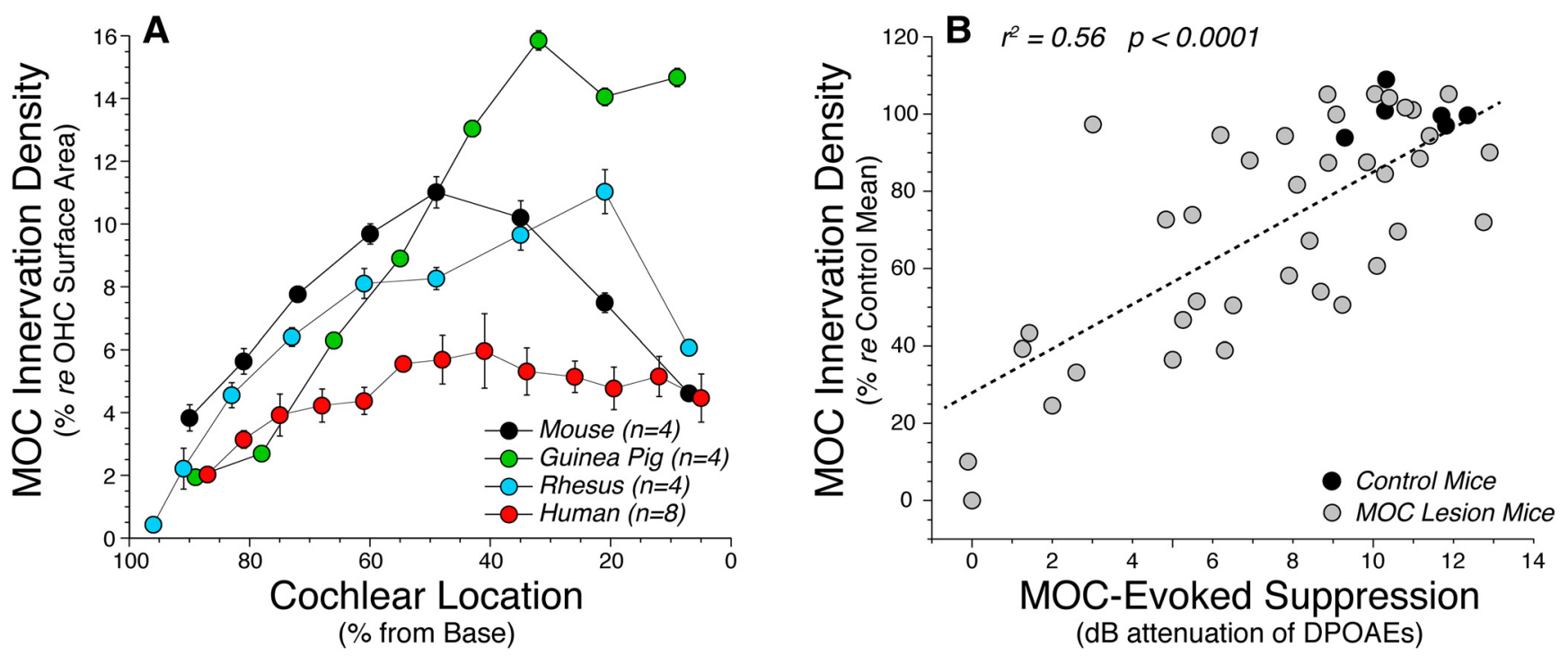

Figure 9. Quantification of $\mathrm{MOC}$ terminal area relative to $\mathrm{OHC}$ surface area in each of four species. $\boldsymbol{A}$, The human data are from the middle-aged group in Figure 5 , expressed relative to $0 \mathrm{HC}$ surface areas extracted from the myosin-immunostaining channel in the same image stacks. Mouse, guinea pig, and rhesus data are from four ears in each species, immunostained, imaged, and analyzed exactly as for the human data and similarly expressed relative to the $\mathrm{OHC}$ surface areas extracted from the same set of image stacks. $\boldsymbol{B}$, To demonstrate the functional validity of the MOC silhouette area metric, we reanalyzed image stacks from a prior study in which MOC feedback strength was measured after partial sectioning of the MOC bundle (Liberman et al., 2014). Each point represents a different case, comparing shock-evoked MOC suppression at the frequency region of maximal effect $(22.6 \mathrm{kHz})$ with the MOC silhouette area in the appropriate cochlear region. The correlation was highly significant $(p<0.001)$.

same antibodies and the same data analysis techniques as were applied to the human ears. In Figure 8, we compare confocal images from the region of maximum MOC density in a human cochlea to those from the other three species. The first-row $\mathrm{OHCs}$ in each stack, as traced from the myosin VIIa channel, are superimposed for comparison. In the mouse and guinea pig, the regular OHC array is easily discernable from the ChAT channel, because almost every $\mathrm{OHC}$ has from two to four ChAT-positive terminals contacting it. A radial MOC gradient is clear in the guinea pig: the first row is more heavily innervated than the second row, and the second row is more heavily innervated than the third row. In the rhesus, the radial gradient is stronger, in that some OHCs in the second row, and many in the third, are not contacted by any MOC terminals. In humans, the innervation appears the patchiest (i.e., many cells in the second and third rows appear uninnervated). Note also that ChAT-positive, tunnel-crossing fibers (Fig. 8, arrowheads) are approximately half as numerous in the human as in the other species: human, 8 fibers for 13 first-row OHCs; rhesus, 15 fibers for 15 OHCs; guinea pig, 13 fibers for 14 OHCs; and mouse, 17 fibers for 16 OHCs.

Quantitative comparison of MOC silhouette areas from the peak-density region, normalized to the number of $\mathrm{OHCs}$ contacted, yields the following mean values for $\mu \mathrm{m}^{2} / \mathrm{OHC}$ ( \pm SEMs): guinea pigs, $34.7( \pm 0.7)$ at $4 \mathrm{kHz}$; monkeys, 21.9 $( \pm 1.3)$ at $2 \mathrm{kHz}$; humans, $17.3( \pm 0.7)$ at $2 \mathrm{kHz}$; and mice, 13.6 $( \pm 0.6)$ at $22.6 \mathrm{kHz}$. By this metric, the peak human projection is $50 \%$ of that in guinea pig and $80 \%$ of that in the rhesus, but slightly larger than that in the mouse. However, this absolutesize metric ignores the enormous size difference of the $\mathrm{OHCs}$ they are driving: in the peak MOC regions, mouse OHCs are $4.8 \mu \mathrm{m}$ in diameter and $25.6 \mu \mathrm{m}$ tall, for a surface area of 387 $\mu \mathrm{m}^{2}$; whereas, human OHCs are $7.8 \mu \mathrm{m}$ in diameter and 38.2 $\mathrm{mm}$ tall, for a surface area of $986 \mu \mathrm{m}^{2}$. In Figure $9 A$, we plot the mean MOC terminal areas, expressed as a percentage of these species- and place-appropriate OHC-area metrics. By this measure, the peak human MOC projection is less than half as large as that in any of the other three species. To address the possibility that the reduced MOC innervation is an artifact of postmortem autolysis, we regressed the MOC silhouette areas against postmortem time and saw no significant correlation ( $r^{2}=0.0024, p=0.82$; data not shown).

Evidence that MOC silhouette areas, as measured here, are functionally significant comes from multiple sources. In cats, MOC silhouette areas versus cochlear frequency, extracted from synaptophysin-immunostained cochleas, are very similar to MOC-elicited threshold elevations in auditory-nerve fibers plotted versus their best frequencies (Liberman et al., 1990, their Fig. 18). In the mouse, counts of surviving MOC terminals after deefferentation surgery are highly correlated with MOC effect size in the same ears $\left(r^{2}=0.58, p<0.0001\right.$; Liberman et al., 2014), measured as the cochlear threshold elevation elicited by electrical stimulation of the MOC bundle. Here, we reanalyzed those mouse data, measuring MOC silhouette areas instead of terminal counts, and found that the correlations were just as significant (Fig. 9B; $r^{2}=0.56, p<0.0001$ ). Not surprisingly, the terminal counts and the silhouette areas were also highly correlated $\left(r^{2}=\right.$ 0.87, $p<0.0001$; data not shown).

Qualitative comparison of LOC innervation densities does not suggest any striking interspecies differences. Figure 10 compares regions of maximum innervation density in human versus mouse. If anything, the human LOC is denser than that in the mouse; for example, the TSB (Fig. 10) is better developed in the human. No quantitative interspecies comparisons were undertaken, because the functional significance of this unmyelinated system is much more poorly understood, and because the peripheral targets, the unmyelinated terminals of auditory-nerve fibers, are too small to be well resolved in these confocal $z$-stacks; thus, no meaningful interspecies normalization to adjust for size differences is possible. 

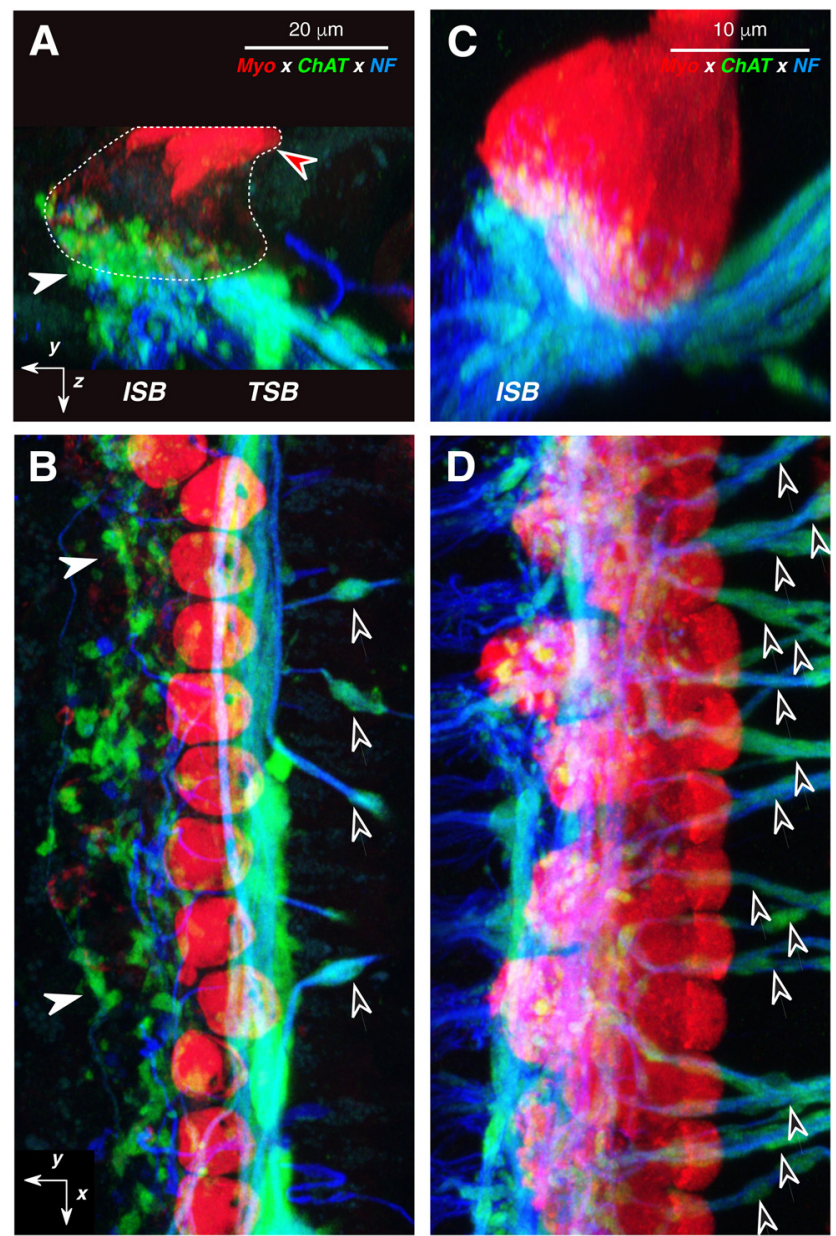

\section{Human $0.35 \mathrm{kHz}$ $\sim 80 \%$ locus}

Figure 10. LOC innervation density in humans is comparable to that in mouse. $\boldsymbol{A}-\boldsymbol{D}$, Confocal projections from the cochlear region with maximal LOC innervation: $0.35 \mathrm{kHz}$ in human $(\boldsymbol{A}$, $\boldsymbol{B})$ vs $8.0 \mathrm{kHz}$ in mouse $(\boldsymbol{C}, \boldsymbol{D})$. The human case patient was a 59 -year-old female. For each species, two views of the same confocal $z$-stack are shown: $\boldsymbol{B}$ and $\boldsymbol{D}$ are maximum projections in the acquisition plane ( $y x$ ), while $\boldsymbol{A}$ and $\boldsymbol{C}$ are maximum projections of the entire $z$-stacks in the $y z$ plane. Orientation arrows in $\boldsymbol{A}$ and $\boldsymbol{B}$ also apply to $\boldsymbol{C}$ and $\boldsymbol{D}$, respectively: the $x$ arrows point along the spiral toward the apex, $y$ arrows point radially away from the modiolus, and $z$ arrows point toward scala tympani. White arrowheads in $\boldsymbol{A}$ and $\boldsymbol{B}$ point to ChAT-positive LOC terminals at the bases of IHCs; black-filled arrowheads in $\boldsymbol{B}$ and $\boldsymbol{D}$ point to the ChAT-positive tunnelcrossing fibers that give rise to MOC terminals. Red-filled arrowhead in $A$ points to $\mathrm{IHC}$ cuticular plates, and the rough outline of $\mathrm{IHCs}$ is shown as a dashed line. The immunostaining key in $\boldsymbol{A}$ applies to all panels. Scale bars in $\boldsymbol{A}$ and $\boldsymbol{C}$ apply to $\boldsymbol{B}$ and $\boldsymbol{D}$, respectively.

\section{Discussion}

\section{OC effects at low versus high frequencies}

All vertebrate hair cell systems, including those in cochlear, vestibular, and lateral-line epithelia, are modulated by a neuronal efferent pathway that projects either to the hair cells themselves or the sensory neurons contacting them (Klinke and Galley, 1974). Although such feedback may be important to several aspects of sensory processing, a common functional role appears to be an automatic gain control, to adjust the dynamic range of the sensor according to the overall level of the stimulus (Guinan, 2010).

Activation of MOC terminals in mammals elevates cochlear thresholds by decreasing intracellular receptor potentials and thereby decreasing the reverse transduction of those electrical signals into mechanical motion, as effected by the motor protein

prestin in OHC membranes (Fuchs and Lauer, 2019). As in other "low-frequency" mammals (Liberman and Gao, 1995; Liberman et al., 2000), the density of MOC terminals peaks in the upper basal turn, $\sim 2-4 \mathrm{kHz}$ in our youngest subjects (Fig. 5), suggesting that this sound-evoked negative feedback is most important for high-frequency stimuli. Similarly, in cats and guinea pigs, MOC innervation peaks in the $5-10 \mathrm{kHz}$ region (Liberman et al., 1990; Liberman and Gao, 1995). Correspondingly, when MOC activity is evoked by electrically stimulating the OC bundle, maximum effects on cochlear thresholds are also seen for fibers tuned to frequencies from 5 to $10 \mathrm{kHz}$ in cats (Guinan and Gifford, 1988).

Such a high-frequency bias is consistent with a functional role of MOC feedback in the control of noise masking, especially at high frequencies (Kawase et al., 1993). One of the mechanisms underlying the decreasing response in the auditory nerve to a transient signal in the presence of continuous noise is vesicle depletion at the IHC synapse (Delgutte, 1990). Since the MOC reflex has an onset time constant of $>100 \mathrm{~ms}$ (Warren and Liberman, 1989; Backus and Guinan, 2006), the negative feedback of the MOC can enhance the auditory-nerve response to a stimulus transient (Winslow and Sachs, 1987) by suppressing the tonic response to, and synaptic fatigue from, a continuous noise. This is particularly important at high frequencies $(>1 \mathrm{kHz})$, where there is diminished ability of auditory-nerve fibers to phase lock to the incoming stimulus (Johnson, 1980), which can provide alternate cues about the stimulus when the signal-to-noise ratio is poor.

In animals and humans, the MOC reflex can be evoked by sound in either ear. Thus, its strength can be assessed by measuring the elevation of ipsilateral thresholds by a contralateral sound (Liberman, 1989). Contralateral sound suppression in humans typically peaks for ipsilateral frequencies $\leq 1 \mathrm{kHz}$ (Kim et al., 2002; Lilaonitkul and Guinan, 2012), which is one to two octaves lower than the MOC innervation peak (Fig. 5). Similarly, in cats, contralateral sound suppression peaks near $2 \mathrm{kHz}$ (Liberman, 1991), which is two octaves lower than the peak MOC density (Liberman et al., 1990) or the peak in shock-evoked threshold elevations (Guinan and Gifford, 1988). In animals, this discrepancy has been ascribed to an anesthesia-related decrease in sound-evoked responses of high-frequency MOC fibers (Liberman, 1988). Since the human assays are performed without anesthesia, an alternative explanation is needed. Perhaps the submaximal reflex activation of MOC neurons at the peak innervation region arises because full activation of this higher frequency "hot spot" requires descending control from higher centers, superimposed on the brainstem reflex (Suthakar and Ryugo, 2017), which might not be activated under the passive listening conditions present during routine testing of MOC reflexes in humans.

With respect to the LOC innervation, less is known about the apical-basal gradient in other mammals. In the cat, LOC terminal density is approximately constant from apex to base, as seen by injection of radioactive tracers into the lateral superior olive (Guinan et al., 1984). In the mouse, cholinergic markers in the IHC area show a relatively uniform projection with a very broad peak in approximately the middle of the cochlea (Maison et al., 2003). Both appear fundamentally different from the human pattern, which shows a strongly decreasing gradient from apex to base (Fig. $7 B$ ). Since the peripheral effects of LOC terminals are more diffuse, difficult to evoke, and more poorly understood (Groff and Liberman, 2003), it is impossible to compare the morphological gradients to any observed physiological gradients. 


\section{OC effects in humans versus other mammals}

The MOC projections we observed in human ears seemed qualitatively less robust than in other common experimental mammals [e.g., guinea pigs (Burgess et al., 1997), cats (Liberman et al., 1990), ferrets (Irving et al., 2011), or mice (Liberman et al., 2014)]. As shown in Figure 8, in the mouse or guinea pig, every $\mathrm{OHC}$ in all three rows is innervated by multiple MOC terminals. In humans, by contrast, few OHCs have multiple MOC contacts, and some have none, especially in the third row. Furthermore, the orthogonal views (Fig. $4 A$ ) show that many ChAT-positive terminals in humans are below the basal pole of the $\mathrm{OHC}$, where they synapse on type II afferent fibers, as is well described in ultrastructural studies of human cochleas (Thiers et al., 2002).

Our quantitative analysis (Fig. 9A) corroborates the qualitative impression (i.e., when normalized to the sizes of the OHCs they contact, peak MOC innervation in humans is $~ 50 \%$ of that in the mouse or rhesus and $<40 \%$ of that in the guinea pig). Such a normalization seems reasonable, as a rough approximation, given that MOC functional effects are mediated via OHC transmembrane voltage, as generated by opening of calcium-activated $\mathrm{K}^{+}$channels. Thus, any MOC-mediated voltage changes in the $\mathrm{OHC}$ will be inversely proportional to the capacitance of the cell, which, in turn, will be proportional to surface area.

These morphological observations suggest that MOC feedback in humans is weaker than in other mammals. Indeed, in normal young adults, OC activation by a moderate-level contralateral sound attenuates ipsilateral probe responses by only 1-2 dB (Collet et al., 1990; Kim et al., 2002; Chambers et al., 2012; Abdala et al., 2014). In contrast, in an unanesthetized mouse, contralateral sound suppression is $\sim 10 \mathrm{~dB}$ (Chambers et al., 2012), and in an unanesthetized guinea pig, ipsilaterally evoked MOC suppression is close to $20 \mathrm{~dB}$ (Boyev et al., 2002). Similarly, in anesthetized cats, electrical stimulation of the MOC system can elevate auditory-nerve thresholds by $\sim 20 \mathrm{~dB}$ (Guinan and Gifford, 1988).

Correspondingly, human studies designed to reveal MOC effects on auditory performance have yielded relatively small effects (for review, see Guinan, 2010). One particularly well controlled study compared hearing-in-noise performance in one ear, with versus without an OC elicitor in the contralateral ear. OC activation made only a small improvement $(10-15 \%)$ on a phoneme identification task in normal subjects. Importantly, this contralateral sound enhancement was absent in five subjects who had undergone an VIIth nerve surgery for intractable vertigo that should cut the OC bundle without disturbing the auditory nerve (Giraud et al., 1997).

In contrast to the sparsity of MOC projections re other mammals, the LOC projection in humans seemed comparably robust, as illustrated by the mouse-human comparison in Figure 10. In both species, the LOC terminals form a cluster that appears to cover the basal pole of the IHCs. The major difference is the prominence of the tunnel spiral bundle in humans, which suggests that LOC fibers may spiral for longer distances in the human than in the mouse. The robustness of the LOC projection in our human material helps to argue against the idea that the paucity of MOC projections is an artifact of postmortem autolysis of ChAT immunoreactivity.

\section{Aging and the OC system}

In a prior report on the same human ears studied here, we showed a significant age-related loss of the myelinated "type I" sensory fibers innervating IHCs, without a significant age-related loss of the unmyelinated type II afferents innervating OHCs (Wu et al.,
2019). Here, we saw parallel patterns in the age-related degeneration of OC projections (i.e., a significant loss of the myelinated MOC pathway) and minimal age-related loss of the unmyelinated LOC pathway. Furthermore, the slope of the age-related MOC decline (7.0\% per decade) was similar to that of the agerelated type I decline (7.8\% per decade). The reasons for a difference in age-related loss of myelinated versus unmyelinated fibers is unclear; however, the myelinated type I fibers are also more vulnerable than unmyelinated type II fibers to other insults such as acoustic trauma or surgical transection of the central axons (Spoendlin, 1975, 1979).

There have been few studies of the age-related decline in the magnitude of MOC reflex strength in humans, especially within the age range for most of our subjects (50-90 years). However, two studies that include a group of subjects $>50$ years of age report a significant decrease in MOC strength, especially at higher frequencies (i.e., 4 vs $1 \mathrm{kHz}$ probe tones; Kim et al., 2002; Lisowska et al., 2014). These results are consistent with the degeneration patterns we observed for MOC innervation density, which also show larger effects in the $4 \mathrm{kHz}$ cochlear region than in the $1 \mathrm{kHz}$ region (Fig. 5). One study reports increased contralateral sound effects in a group of older subjects (63-73 years of age vs younger groups of $13-17,19-27$, or $40-58$ years of age), but suggests that the results could be confounded by enhanced activation of the middle-ear muscle reflex in the older group (Abdala et al., 2014).

Thus, an age-related decline in MOC innervation density, especially in the high-frequency regions where energy from many consonants is concentrated, could also contribute to the reduced hearing-in-noise abilities that are a classic feature of age-related hearing loss. However, equally important to any age-related performance decrements is the loss of the OHCs themselves, without which the MOC system is unable to perform any adjustment of the dynamic range.

\section{References}

Abdala C, Dhar S, Ahmadi M, Luo P (2014) Aging of the medial olivocochlear reflex and associations with speech perception. J Acoust Soc Am 135:754-765.

Backus BC, Guinan JJ Jr (2006) Time-course of the human medial olivocochlear reflex. J Acoust Soc Am 119:2889-2904.

Boyev KP, Liberman MC, Brown MC (2002) Effects of anesthesia on efferent-mediated adaptation of the DPOAE. J Assoc Res Otolaryngol 3:362-373.

Burgess BJ, Adams JC, Nadol JB Jr (1997) Morphologic evidence for innervation of Deiters' and Hensen's cells in the guinea pig. Hear Res 108: $74-82$.

Chambers AR, Hancock KE, Maison SF, Liberman MC, Polley DB (2012) Sound-evoked olivocochlear activation in unanesthetized mice. J Assoc Res Otolaryngol 13:209-217.

Collet L, Kemp DT, Veuillet E, Duclaux R, Moulin A, Morgon A (1990) Effect of contralateral auditory stimuli on active cochlear micromechanical properties in human subjects. Hear Res 43:251-261.

Darrow KN, Maison SF, Liberman MC (2006) Cochlear efferent feedback balances interaural sensitivity. Nat Neurosci 9:1474-1476.

Darrow KN, Maison SF, Liberman MC (2007) Selective removal of lateral olivocochlear efferents increases vulnerability to acute acoustic injury. J Neurophysiol 97:1775-1785.

Delgutte B (1990) Physiological mechanisms of psychophysical masking: observations from auditory-nerve fibers. J Acoust Soc Am 87:791-809.

Fuchs PA, Lauer AM (2019) Efferent inhibition of the cochlea. Cold Spring Harb Perspect Med 9:a033530.

Giraud AL, Garnier S, Micheyl C, Lina G, Chays A, Chéry-Croze S (1997) Auditory efferents involved in speech-in-noise intelligibility. Neuroreport 8:1779-1783.

Gordon-Salant S (2005) Hearing loss and aging: new research findings and clinical implications. J Rehabil Res Dev 42:9-24. 
Greenwood DD (1990) A cochlear frequency-position function for several species-29 years later. J Acoust Soc Am 87:2592-2605.

Groff JA, Liberman MC (2003) Modulation of cochlear afferent response by the lateral olivocochlear system: activation via electrical stimulation of the inferior colliculus. J Neurophysiol 90:3178-3200.

Guinan JJ Jr (2010) Cochlear efferent innervation and function. Curr Opin Otolaryngol Head Neck Surg 18:447-453.

Guinan JJ Jr, Warr WB, Norris BE (1983) Differential olivocochlear projections from lateral versus medial zones of the superior olivary complex. J Comp Neurol 221:358-370.

Guinan JJ Jr, Warr WB, Norris BE (1984) Topographic organization of the olivocochlear projections from the lateral and medial zones of the superior olivary complex. J Comp Neurol 226:21-27.

Guinan JJ Jr, Gifford ML (1988) Effects of electrical stimulation of efferent olivocochlear neurons on cat auditory-nerve fibers. III. Tuning curves and thresholds at CF. Hear Res 37:29-45.

Irving S, Moore DR, Liberman MC, Sumner CJ (2011) Olivocochlear efferent control in sound localization and experience-dependent learning. J Neurosci 31:2493-2501.

Ishii T, Murakami Y, Balogh K Jr (1967) Acetylcholinesterase activity in the efferent nerve fibers of the human inner ear. Ann Otol Rhinol Laryngol 76:69-82.

Jacobson M, Kim S, Romney J, Zhu X, Frisina RD (2003) Contralateral suppression of distortion-product otoacoustic emissions declines with age: a comparison of findings in CBA mice with human listeners. Laryngoscope 113:1707-1713.

Johnson DH (1980) The relationship between spike rate and synchrony in responses of auditory-nerve fibers to single tones. J Acoust Soc Am 68:1115-1122.

Kawase T, Delgutte B, Liberman MC (1993) Antimasking effects of the olivocochlear reflex. II. Enhancement of auditory-nerve response to masked tones. J Neurophysiol 70:2533-2549.

Kim S, Frisina DR, Frisina RD (2002) Effects of age on contralateral suppression of distortion product otoacoustic emissions in human listeners with normal hearing. Audiol Neurootol 7:348-357.

Klinke R, Galley N (1974) Efferent innervation of vestibular and auditory receptors. Physiol Rev 54:316-357.

Konomi U, Kanotra S, James AL, Harrison RV (2014) Age related changes to the dynamics of contralateral DPOAE suppression in human subjects. J Otolaryngol Head Neck Surg 43:15.

Kujawa SG, Liberman MC (1997) Conditioning-related protection from acoustic injury: effects of chronic deefferentation and sham surgery. J Neurophysiol 78:3095-3106.

Liberman MC (1980) Efferent synapses in the inner hair cell area of the cat cochlea: an electron microscopic study of serial sections. Hear Res 3:189-204.

Liberman MC (1988) Response properties of cochlear efferent neurons: monaural vs. binaural stimulation and the effects of noise. J Neurophysiol 60:1779-1798.

Liberman MC (1989) Rapid assessment of sound-evoked olivocochlear feedback: suppression of compound action potentials by contralateral sound. Hear Res 38:47-56.

Liberman MC (1991) The olivocochlear efferent bundle and susceptibility of the inner ear to acoustic injury. J Neurophysiol 65:123-132.

Liberman MC, Brown MC (1986) Physiology and anatomy of single olivocochlear neurons in the cat. Hear Res 24:17-36.

Liberman MC, Gao WY (1995) Chronic cochlear de-efferentation and susceptibility to permanent acoustic injury. Hear Res 90:158-168.

Liberman MC, Dodds LW, Pierce S (1990) Afferent and efferent innervation of the cat cochlea: quantitative analysis with light and electron microscopy. J Comp Neurol 301:443-460.

Liberman MC, O'Grady DF, Dodds LW, McGee J, Walsh EJ (2000) Afferent innervation of outer and inner hair cells is normal in neonatally deefferented cats. J Comp Neurol 423:132-139.

Liberman MC, Liberman LD, Maison SF (2014) Efferent feedback slows cochlear aging. J Neurosci 34:4599-4607.

Lilaonitkul W, Guinan JJ Jr (2012) Frequency tuning of medial-olivo- cochlear-efferent acoustic reflexes in humans as functions of probe frequency. J Neurophysiol 107:1598-1611.

Lisowska G, Namyslowski G, Orecka B, Misiolek M (2014) Influence of aging on medial olivocochlear system function. Clin Interv Aging 9:901-914.

Maison SF, Adams JC, Liberman MC (2003) Olivocochlear innervation in the mouse: immunocytochemical maps, crossed versus uncrossed contributions, and transmitter colocalization. J Comp Neurol 455:406-416.

Moore JK, Simmons DD, Guan Y (1999) The human olivocochlear system: organization and development. Audiol Neurootol 4:311-325.

Nadol JB Jr (1983a) Serial section reconstruction of the neural poles of hair cells in the human organ of Corti. I. Inner hair cells. Laryngoscope 93:599-614.

Nadol JB Jr (1983b) Serial section reconstruction of the neural poles of hair cells in the human organ of Corti. II. Outer hair cells. Laryngoscope 93:780-791.

Nomura Y (1976) Nerve fibers in the human organ of Corti. Acta Otolaryngol 82:317-324.

Rajan R (1988) Effect of electrical stimulation of the crossed olivocochlear bundle on temporary threshold shifts in auditory sensitivity. I. Dependence on electrical stimulation parameters. J Neurophysiol 60:549-568.

Russell IJ, Murugasu E (1997) Medial efferent inhibition suppresses basilar membrane responses to near characteristic frequency tones of moderate to high intensities. J Acoust Soc Am 102:1734-1738.

Schrott-Fischer A, Egg G, Kong WJ, Renard N, Eybalin M (1994) Immunocytochemical detection of choline acetyltransferase in the human organ of Corti. Hear Res 78:149-157.

Schrott-Fischer A, Kammen-Jolly K, Scholtz A, Rask-Andersen H, Glueckert R, Eybalin M (2007) Efferent neurotransmitters in the human cochlea and vestibule. Acta Otolaryngol 127:13-19.

Schuknecht HF (1993) Pathology of the ear, Ed 2. Baltimore: Lea \& Febiger.

Spoendlin H (1975) Retrograde degeneration of the cochlear nerve. Acta Otolaryngol 79:266-275.

Spoendlin H (1979) Neural connections of the outer haircell system. Acta Otolaryngol 87:381-387.

Suthakar K, Ryugo DK (2017) Descending projections from the inferior colliculus to medial olivocochlear efferents: mice with normal hearing, early onset hearing loss, and congenital deafness. Hear Res 343:34-49.

Taberner AM, Liberman MC (2005) Response properties of single auditory nerve fibers in the mouse. J Neurophysiol 93:557-569.

Thiers FA, Burgess BJ, Nadol JB Jr (2002) Axodendritic and dendrodendritic synapses within outer spiral bundles in a human. Hear Res 164:97-104.

Tsuji J, Liberman MC (1997) Intracellular labeling of auditory nerve fibers in guinea pig: central and peripheral projections. J Comp Neurol 381: $188-202$.

Valero MD, Burton JA, Hauser SN, Hackett TA, Ramachandran R, Liberman MC (2017) Noise-induced cochlear synaptopathy in rhesus monkeys (Macaca mulatta). Hear Res 353:213-223.

Walsh EJ, McGee J, McFadden SL, Liberman MC (1998) Long-term effects of sectioning the olivocochlear bundle in neonatal cats. J Neurosci 18: 3859-3869.

Warren EH 3rd, Liberman MC (1989) Effects of contralateral sound on auditory-nerve responses. I. Contributions of cochlear efferents. Hear Res 37:89-104

Wiederhold ML, Kiang NY (1970) Effects of electric stimulation of the crossed olivocochlear bundle on single auditory-nerve fibers in the cat. J Acoust Soc Am 48:950-965.

Winslow RL, Sachs MB (1987) Effect of electrical stimulation of the crossed olivocochlear bundle on auditory nerve response to tones in noise. J Neurophysiol 57:1002-1021.

Wu PZ, Liberman LD, Bennett K, de Gruttola V, O’Malley JT, Liberman MC (2019) Primary neural degeneration in the human cochlea: evidence for hidden hearing loss in the aging ear. Neuroscience 407:8-20.

Yin Y, Liberman LD, Maison SF, Liberman MC (2014) Olivocochlear innervation maintains the normal modiolar-pillar and habenular-cuticular gradients in cochlear synaptic morphology. J Assoc Res Otolaryngol 15: $571-583$. 